\title{
Fundamental effects of PD-I antibody on the body: a brief report
}

This article was published in the following Dove Press journal:

OncoTargets and Therapy

7 July 2016

Number of times this article has been viewed

\author{
Wei Wang' \\ Bing Liu' \\ Guangxian Liu' \\ Wanfeng Guo² \\ 'Cancer Therapy Center, ${ }^{2}$ Department \\ of Healthcare and Medicine, Affiliated \\ Hospital of the Academy of Military \\ Medical Sciences, Beijing, \\ People's Republic of China
}

\begin{abstract}
We report the case of a 61-year-old man with squamous cell carcinoma of the left lung and mediastinal lymph node metastases who received nivolumab and experienced a better antitumor effect than expected but died soon afterward. A clinical analysis showed that the grades of the treatment-related events did not cause death. The immunological impact of this treatment on his body, especially at his age and with concurrent infection, possibly augmented by the blockade of the PD-1/PD-L1 signaling pathway, is proposed to be one of the key factors for his death. Consequently, we suggest that the cellular immune status and the clinical characteristics of patients, especially the symptoms like concurrent infection, should be considered in the design of clinical protocol of such a kind of therapy.
\end{abstract}

Keywords: lung cancer, PD-1, nivolumab, immunotherapy, lung infection, immunosuppression

\section{Introduction}

Recently, an immune checkpoint blockade strategy has been successfully employed for the treatment of cancers in clinics. ${ }^{1,2}$ Preliminary data have proved the potential of this strategy in cancer immunotherapy. The mechanism of the therapy is to block the signaling pathway of PD-1/PD-L1 or B7/CTLA-4 by using humanized antibodies against PD-1 and CTLA-4 on immune cells or PD-L1 on tumor cells, so as to elevate the antitumor activity of immune cells toward tumor cells. ${ }^{3}$ To date, PD-1 antibodies such as nivolumab or pembrolizumab and CTLA-4 antibodies such as ipilimumab have been approved for treatment of cancers by the US Food and Drug Administration, and a series of clinical trials are anticipated globally. ${ }^{4,5}$ However, the undesirable effects of these drugs on the body, especially on the immune system, still need further evaluation.

\section{Case report}

A 61-year-old man with a history of smoking was diagnosed with left lung cancer and mediastinal lymph node metastases. From May 2013 to December 2014, the patient was treated with erlotinib hydrochloride targeted therapy, two regimens of chemotherapy, and thoracic radiation therapy at a dose of 4,020 cGy/5 f/1 wk. In March 2015, disease progression was observed in the left lung and humerus lesions; thus, radiotherapy was administered at a dose of 3,600 cGy/12 f/3 wk. In July 2015, he was transferred to our hospital. Further examinations, including nuclear magnetic resonance imaging of his head, pathological examination, and positron emission tomography-computed tomography were conducted, showing multiple metastases in his brain, lymph nodes, liver, spleen, adrenal gland, and humerus (Figure 1A-E). A pathological analysis of the liver biopsy revealed a moderate-low differentiation squamous cell carcinoma 

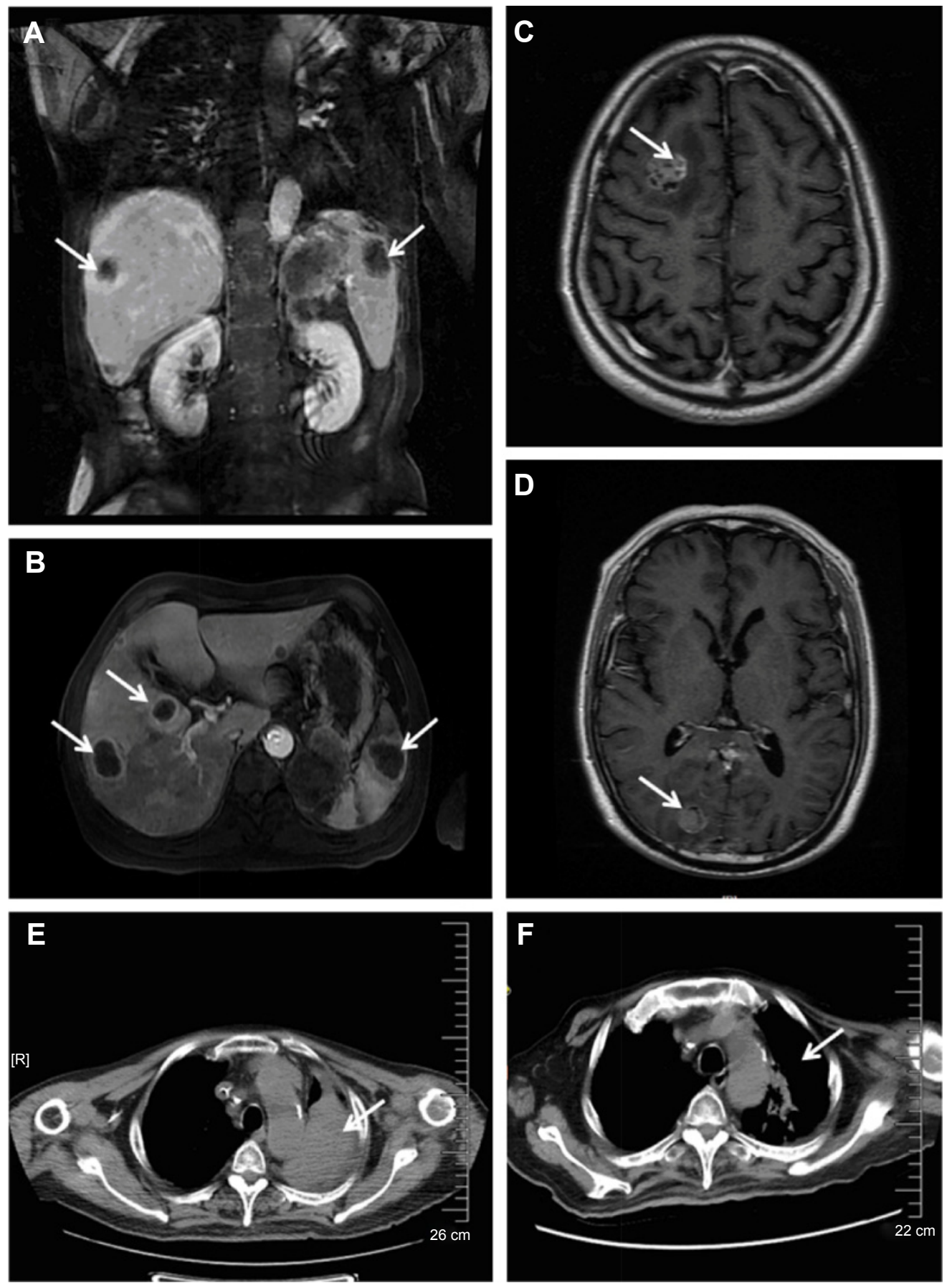

Figure I Imaging materials of the patient during the anti-PD-I treatment.

Notes: MRI showed multiple metastases occurred in the liver $(\mathbf{A})$, spleen $(\mathbf{B})$, and brain $(\mathbf{C}$ and $\mathbf{D})$. A chest $\mathrm{CT}$ scan of the thorax showed lung lesion (E) before and (F) $\sim 5$ days after three cycles of nivolumab treatment. Arrows indicate the lesions.

Abbreviations: MRI, magnetic resonance imaging; CT, computed tomography; PD, programmed death.

with necrosis. The tumor exhibited the following immunohistochemical characteristics: CK5(+), p40(+), CK7(-), CK20(-), TTF-1(-), Napsin A(-), CDX2(-), CK19(+), and GPC3(-).

After integrated consideration and informed consent, we concluded that palliative treatment was appropriate. Anti-PD-1 antibody was a suitable choice. ${ }^{1}$ At the request of the patient for further treatment, we administered nivolumab, in an effort to control the advanced disease and prolong survival and quality of life. Nivolumab was administered at $3 \mathrm{mg} / \mathrm{kg}$ for 1 hour, once every 2 weeks, as a total of three infusions from August 6, 2015, to September 4, 2015. ${ }^{6,7}$ On the second day after the first infusion, the patient developed an intermittent fever, with a maximum body temperature of $39.6^{\circ} \mathrm{C}$, and the routine 
A

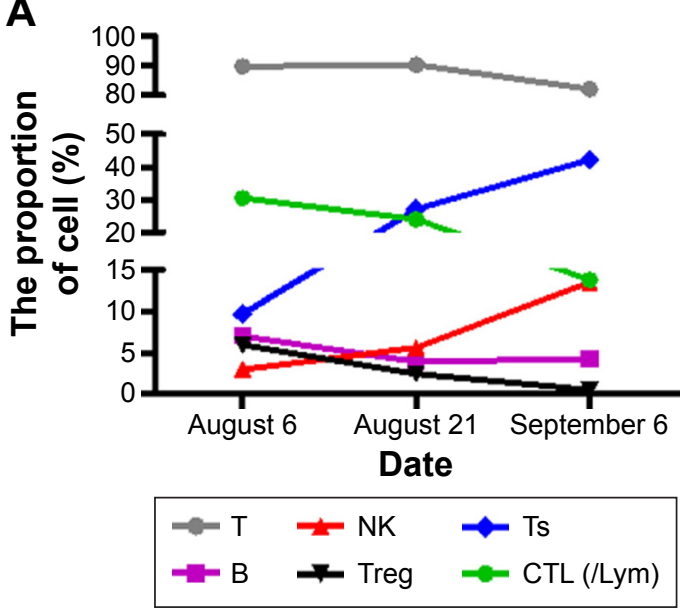

B

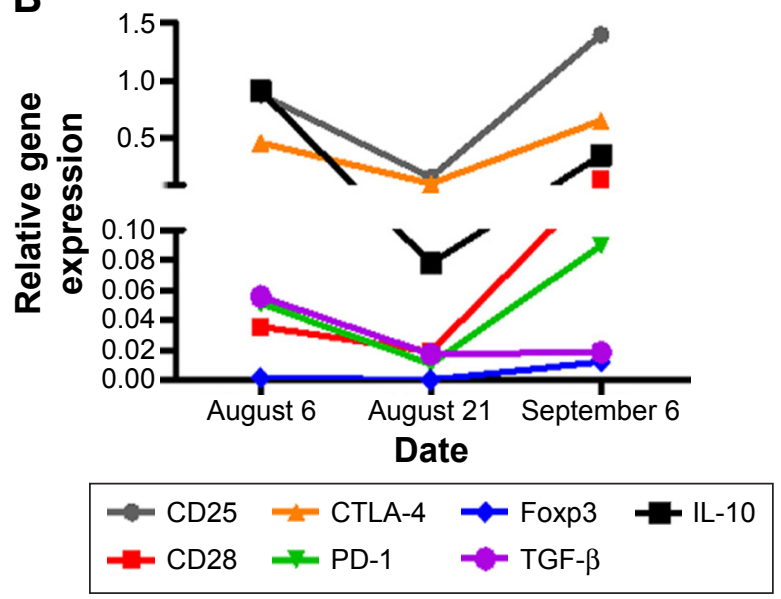

Figure 2 Monitoring of immune status during the anti-PD-I treatment.

Notes: (A) Analysis of the Lym proportions, eg, T, B, NK, Treg, Ts, and CTL, in the peripheral blood. (B) The expression analysis of functional immune molecules, including CD25, CD28, CTLA-4, PD-I, Foxp3, TGF- $\beta$, and IL-I0.

Abbreviations: Lym, lymphocyte; NK, natural killer; Treg, regulatory T; Ts, suppressor T Lym; CTL, cytotoxic T Lym; CTLA, cytotoxic T lymphocyte-associated antigen; PD, programmed death; TGF, transforming growth factor; IL- I0, interleukin- 10.

blood examination showed that his white blood cell count had increased to $15.72 \times 10^{9} / \mathrm{L}$ with $96.8 \%$ neutrophil granulocytes and the C-reactive protein had elevated to $156 \mathrm{mg} / \mathrm{L}$, which indicated a severe infection (grade 3, National Cancer Institute Common Terminology Criteria for Adverse Events 3.0). Bacterial and fungal cultures of sputum showed Acinetobacter baumannii and Candida albicans infections. Then, meropenem ( $0.5 \mathrm{~g} \mathrm{q} 8 \mathrm{~h})$ and fluconazole (200 $\mathrm{mg} \mathrm{qd})$ were administered intravenously on August 8, according to drug sensitivity tests. After treatment with antibiotics, further bacterial and fungal cultures of sputum were conducted on August 16 and were negative for A. baumannii and C. albicans. The white blood cell count had decreased to $9.0 \times 10^{9} / \mathrm{L}$ and the C-reactive protein had dropped to $65 \mathrm{mg} / \mathrm{L}$, suggesting that the infection was controlled. As a result, meropenem and fluconazole infusions were discontinued. Subsequently, the patient had a fever every other day, which was relieved each time by administration of dexamethasone. He experienced mild hemoptysis of $\sim 10 \mathrm{~mL}$ of blood on September 11 and slightly blood-stained sputum on the following 3 days. We gave him hemostasis and coagulation. Moreover, the patient had a progressive aggravation of thrombocytopenia (platelet count dropped to $28 \times 10^{9} / \mathrm{L}$ ), although it was normal before the therapy. Chills, cough, sputum expectoration, and shortness of breath were observed after each antibody infusion. These symptoms were alleviated through anti-infection therapy with meropenem and fluconazole, as well as dexamethasone. A chest computed tomography examination revealed that the treatment was effective and the lung lesions had shrunk after the therapy (Figure 1F), but the patient passed away on September 26 without other severe symptoms.
Cellular immunity was monitored during the treatment. The proportion of lymphocyte (Lym) subpopulations, including $\mathrm{T}, \mathrm{B}$, natural killer (NK), regulatory $\mathrm{T}$ (Treg), cytotoxic T Lym (CTL; $\mathrm{CD}^{+} \mathrm{CD}^{+} \mathrm{CD} 28^{+}$), and suppressor $\mathrm{T}$ Lym (Ts; $\left.\mathrm{CD}^{+}{ }^{+} \mathrm{CD} 8^{+} \mathrm{CD} 28^{-}\right),{ }^{8,9}$ and the expression of several immunoregulatory molecules (inhibition and activation), including CD25, CD28, CTLA-4, PD-1, Foxp3, TGF- $\beta$, and IL-10 in the peripheral blood, were analyzed (Figure 2A and B). Of the Lym proportions, NK cells and Ts were significantly upregulated and CTLs were moderately downregulated, whereas the other Lyms did not vary notably during the observation. The expression of CD25, CD28, CTLA-4, PD-1, and IL-10, but not of Foxp3 and TGF- $\beta$, reduced after the first infusion and then rebounded sharply after the second infusion. The Human Ethics Committee of the Affiliated Hospital of the Academy of Military Medical Sciences approved the case report and the patient provided written informed consent.

\section{Discussion}

Preliminary clinical studies of monoclonal antibodies targeting PD-1 and PD-L1 presented striking results but exhibited moderate side effects. ${ }^{10,11}$ Topalian et al ${ }^{12}$ observed drug-related grade 3 or 4 toxic effects, including pneumonitis with findings ranging from isolated radiographic abnormalities to progressive and diffuse infiltrates associated with clinical symptoms in $14 \%$ of patients who received anti-PD-1 antibody and suggested that the therapy could be delivered in an outpatient setting with minimal supportive care. Brahmer et al reported adverse events, of any grade, in 188 of 207 patients (91\%) who received anti-PD-L1 
antibody. The most common drug-related adverse events were fatigue, infusion reactions, diarrhea, arthralgia, rash, nausea, pruritus, and headache. Most events were of low grade, with treatment-related grade 3 or 4 events noted in 19 of 207 patients (9\%). Potential immune-related side effects were observed in 81 of 207 patients (39\%), including rash, hypothyroidism, and hepatitis, and there was one case each of sarcoidosis, endophthalmitis, diabetes mellitus, and myasthenia gravis. ${ }^{13}$

In this case, an obvious antitumor effect was observed after the first infusion of anti-PD-1 antibody and the tumor shrunk significantly after the third antibody infusion (Figure 1E and F), but the patient died soon afterward without any other severe symptoms except the common side complications such as chills, cough, sputum, shortness of breath, and intermittent fever related to antibody infusion, as well as infection and thrombocytopenia. Although we were not able to differentiate factors influencing the clinical symptoms and outcomes from those of others, special attention should be paid to the systematic and fundamental immune effects of the treatment on the body. In the present case, analysis of the cellular immune function showed that, from the first infusion, B, CTLs $\left(\mathrm{CD}^{+} \mathrm{CD} 8^{+} \mathrm{CD} 28^{+}\right)$, and Treg populations gradually declined, whereas NK cells clearly increased and the Ts population $\left(\mathrm{CD} 3{ }^{+} \mathrm{CD} 8^{+} \mathrm{CD} 28^{-}\right)$ became sharply elevated. The shift in the Lym subpopulations of special interest was the Ts and NK upregulation and the $\mathrm{T}$ and $\mathrm{B}$ downregulation, which indicated an obvious immunosuppressive regulation and might represent a feedback immunoregulation mediated by blockade of the PD-1/PD-L1 signaling pathway by the anti-PD-1 antibody and the immune response to the bacterial infection. In addition, evaluation of the immune regulatory signals revealed that the expression of CD25, CD28, CTLA-4, PD-1, and IL-10, but not of TGF- $\beta$ and Foxp3, became strikingly upregulated after the second infusion, though all of them had been clearly downregulated after the first infusion, which also indicated an immunosuppressive regulation and further supported our inference above. The immunomodulation mechanism mediated by the antibody infusion and its role in the death are still unknown and thus remain to be further investigated.

Thrombocytopenia was one of the obvious complications in this case. Nugent et al has reported that autoimmune disease may impair megakaryocyte platelet production and accelerate platelet destruction, which cause immune thrombocytopenia. ${ }^{14}$ Cytotoxic effects of $\mathrm{CD}^{+} \mathrm{T}$ Lym are also thought to cause thrombocytopenia by impairing megakaryocytopoiesis. ${ }^{15}$ The immunological effect could be one of the key factors for the inhibition of platelet production. In this case, blockade of PD-1/PD-L1 naturally elevated immune function and also increased autologous immune response. Moreover, the intermittent fever, which can be attributed to the immunological side effects of the treatment and was relieved by the administration of dexamethasone following control of the initial infection, might be another cause of the damage to platelets. ${ }^{16,17}$

Another important complication in this patient was the infection, as pneumonitis is reported to be one of the general immune-mediated toxic effects with high mortality related to the use of antibodies against PD-1. Topalian et al ${ }^{12}$ reported $3 \%$ (nine of 296 patients) drug-related pneumonitis with $1 \%$ (three cases) grade 3 or 4 pneumonitis and three drug-related deaths $(1 \%)$ due to pneumonitis (two in patients with nonsmall-cell lung cancer and one in a patient with colorectal cancer) in a Phase I trial. Nishino et $\mathrm{al}^{18}$ reported three cases with anti-PD-1-related pneumonitis, one of whom died 4 weeks after the diagnosis of pneumonitis. The onset of their pneumonitis occurred 7.4-24.3 months after the initiation of the therapy. The only difference in our case was that the infection occurred immediately after the infusion of antiPD-1 antibody. In fact, we had paid much attention on the pneumonitis, but the bacterial infection has been controlled by the treatment with antibiotics and we believed that the grade of patient's lung infection would not lead to severe complication or death. However, another possibility exists that the immune response modulated by nivolumab might have been augmented by the concurrent infection that might have resulted in death, for which much attention should be paid by a physician when anti-PD-1 antibody is administered, especially for patients with concurrent infections and who are old. In addition, the immunological damage caused by such treatment remains unknown and it should be considered in the design of following treatment.

\section{Conclusion}

We suggest that the cellular immune status and the clinical characteristics of patients need to be considered in the design of clinical protocols for immune checkpoint blockade therapy, especially in those with concurrent infections and in older patients. In the case discussed here, if the antibody had been discontinued at the second infusion or if further immunomodulation therapy had been received before further antibody infusions, using the antibodies as an immunomodulator rather than as a drug, the outcome might have been altered. ${ }^{19}$ 


\section{Acknowledgments}

Grant Support:This study was supported by the National Natural Science Foundation (No 81372251).

\section{Disclosure}

The authors report no conflicts of interest in this work.

\section{References}

1. Brahmer J, Reckamp KL, Baas P, et al. Nivolumab versus docetaxel in advanced squamous-cell non-small-cell lung cancer. NEngl J Med. 2015; 373(2):123-135.

2. Rizvi NA, Mazieres J, Planchard D, et al. Activity and safety of nivolumab, an anti-PD-1 immune checkpoint inhibitor, for patients with advanced, refractory squamous non-small-cell lung cancer (CheckMate 063): a phase 2, single-arm trial. Lancet Oncol. 2015;16(3):257-265.

3. Pardoll DM. The blockade of immune checkpoints in cancer immunotherapy. Nat Rev Cancer. 2012;12(4):252-264.

4. Brahmer JR, Drake CG, Wollner I, et al. Phase I study of single-agent anti-programmed death-1 (MDX-1106) in refractory solid tumors: safety, clinical activity, pharmacodynamics, and immunologic correlates. J Clin Oncol. 2010;28(19):3167-3175.

5. Lynch TJ, Bondarenko I, Luft A, et al. Ipilimumab in combination with paclitaxel and carboplatin as first-line treatment in stage IIIB/IV non-small-cell lung cancer: results from a randomized, double-blind, multicenter phase II study. J Clin Oncol. 2012;30(17):2046-2054.

6. Keating GM. Nivolumab: a review in advanced squamous non-small cell lung cancer. Drugs. 2015;75(16):1925-1934.

7. Guibert N, Mazieres J. Nivolumab for treating non-small cell lung cancer. Expert Opin Biol Ther. 2015;15(12):1789-1797.

8. Haubeck HD, Minkenberg I, Kolsch E. Analysis of T suppressor cellmediated tumor escape mechanisms is facilitated by the selective in vitro activation of tumor-specific Ts cells. Haematol Blood Transfus. 1989;32: $260-262$.
9. Nociari MM, Telford W, Russo C. Postthymic development of CD28CD8+ T cell subset: age-associated expansion and shift from memory to naive phenotype. J Immunol. 1999;162(6):3327-3335.

10. Gangadhar TC, Salama AK. Clinical applications of PD-1-based therapy: a focus on pembrolizumab (MK-3475) in the management of melanoma and other tumor types. Onco Targets Ther. 2015;8: 929-937.

11. Villadolid J, Amin A. Immune checkpoint inhibitors in clinical practice: update on management of immune-related toxicities. Transl Lung Cancer Res. 2015;4(5):560-575.

12. Topalian SL, Hodi FS, Brahmer JR, et al. Safety, activity, and immune correlates of anti-PD-1 antibody in cancer. N Engl J Med. 2012;366(26): 2443-2454.

13. Brahmer JR, Tykodi SS, Chow LQ, et al. Safety and activity of antiPD-L1 antibody in patients with advanced cancer. $N$ Engl $J$ Med. 2012;366(26):2455-2465.

14. Nugent D, McMillan R, Nichol JL, Slichter SJ. Pathogenesis of chronic immune thrombocytopenia: increased platelet destruction and/or decreased platelet production. Br J Haematol. 2009;146(6):585-596.

15. Olsson B, Andersson PO, Jernås M, et al. T-cell-mediated cytotoxicity toward platelets in chronic idiopathic thrombocytopenic purpura. Nat Med. 2003;9(9):1123-1124.

16. Jaramillo Melba C, Frye Jennifer B, Crapo JD, et al. Increased manganese superoxide dismutase expression or treatment with a manganese porphyrin potentiates dexamethasone-induced apoptosis in lymphoma cells. Cancer Res. 2009;69(13):5450-5457.

17. Smith LK, Cidlowski JA. Glucocorticoid-induced apoptosis of healthy and malignant lymphocytes. Prog Brain Res. 2010;182:1-30.

18. Nishino M, Sholl LM, Hodi FS. Anti-PD-1-related pneumonitis during cancer immunotherapy. $N$ Engl J Med. 2015;373(3):288-290.

19. Liu GX, Yang WW, Guo M, et al. Effective modulation of CD4 $4^{+} \mathrm{CD} 25^{+}$ highregulatory $\mathrm{T}$ and $\mathrm{NK}$ cells in malignant patients by combination of interferon- $\alpha$ and interleukin-2. Cancer Immunol Immunother. 2012;61(12):2357-2366.
OncoTargets and Therapy

\section{Publish your work in this journal}

OncoTargets and Therapy is an international, peer-reviewed, open access journal focusing on the pathological basis of all cancers, potential targets for therapy and treatment protocols employed to improve the management of cancer patients. The journal also focuses on the impact of management programs and new therapeutic agents and protocols on

\section{Dovepress}

patient perspectives such as quality of life, adherence and satisfaction. The manuscript management system is completely online and includes a very quick and fair peer-review system, which is all easy to use. Visit http://www.dovepress.com/testimonials.php to read real quotes from published authors. 